\title{
Correction to: Pre-operative templating in THA. Part II: a CT-based strategy to correct architectural hip deformities
}

\author{
Hideo Kobayashi ${ }^{1} \cdot$ Alexandre Cech $^{2} \cdot$ Masanori Kase $^{3} \cdot$ Geert Pagenstert $^{4,5,6} \cdot$ Yannick Carrillon $^{2}$. \\ Padhraig F. O'Loughlin ${ }^{2,7} \cdot$ Hugo Bothorel $^{8} \odot$ - Tarik Aït-Si-Selmi ${ }^{2,9} \cdot$ Michel P. Bonnin $^{2,9}$
}

Published online: 5 September 2020

(c) Springer-Verlag GmbH Germany, part of Springer Nature 2020

\section{Correction to: \\ Archives of Orthopaedic and Trauma Surgery (2020) 140:551-562 \\ https://doi.org/10.1007/s00402-020-03341-6}

The original version of this article unfortunately contained a mistake. The surname of one of the authors was misspelled as Geert Pagenstart instead of the correct name which should be Geert Pagenstert.

Publisher's Note Springer Nature remains neutral with regard to jurisdictional claims in published maps and institutional affiliations.

The original article can be found online at https://doi.org/10.1007/ s00402-020-03341-6.

Hugo Bothorel

journals@ resurg.com

1 Department of Orthopaedic Surgery, Juntendo University, Bunkyo-ku, Tokyo, Japan

2 Ramsay Santé, Hôpital Privé Jean Mermoz, Centre Orthopédique Santy, Lyon, France

3 Department of Orthopaedic Surgery, Nissan Tamagawa Hospital, Setagayaku, Tokyo, Japan

4 Department of Clinical Research, University of Basel, Basel, Switzerland

5 Clarahof Clinic of Orthopaedic Surgery, Merian-Iselin-Hospital Swiss Olympic Medical Center, Basel, Switzerland

6 Knee Institute Basel, Basel, Switzerland

7 Cork University Hospital, South Infirmary, Victoria University Hospital, Mater Private Cork, Cork, Ireland

8 ReSurg SA, Rue Saint-Jean 22, 1260 Nyon, Switzerland

9 Artro Institute, Lyon, France 